\title{
Performance Optimization for State Machine Replication Based on Application Semantics: A Review
}

\author{
Wenbing Zhao* \\ Department of Electrical Engineering and Computer Science, Cleveland State University, \\ 2121 Euclid Avenue, Cleveland, OH 44115, USA
}

\begin{abstract}
The pervasiveness of cloud-based services has significantly increased the demand for highly dependable systems. State machine replication is a powerful way of constructing highly dependable systems. However, state machine replication requires replicas to run deterministically and to process requests sequentially according to a total order. In this article, we review various techniques that have been used to engineer fault tolerance systems for better performance. Common to most such techniques is the customization of fault tolerance mechanisms based on the application semantics. By incorporating application semantics into fault tolerance design, we could enable concurrent processing of requests, reduce the frequency of distributed agreement operations, and control application nondeterminism. We start this review by making a case for considering application semantics for state machine replication. We then present a classification of various approaches to enhancing the performance of fault tolerance systems. This is followed by the description of various fault tolerance mechanisms. We conclude this article by outlining potential future research in high performance fault tolerance computing.
\end{abstract}

Keywords: Application Semantics, State Machine Replication, Fault Tolerance, Application Nondeterminism, Byzantine Agreement, Conflict-Free Replicated Data Types, Operational Transformation

\footnotetext{
${ }^{*}$ Corresponding author

Email address: wenbing@ieee.org (Wenbing Zhao)
} 


\section{Introduction}

The pervasiveness of cloud-based services has significantly increased the dependability expectations of many computer systems not only from business partners, but also from many millions of end-users. Such systems must be made resilient against various hardware failures and possibly against cyber attacks as well. State machine replication has shown to be an effective technique to help achieve this goal (Zhao, 2014b). Highly efficient fault tolerance algorithms have been developed to tolerate both crash faults and Byzantine faults, the most seminal of which are Paxos (Lamport, 1998, 2001) and PBFT (Castro and Liskov, 2002). However, state machine replication requires replicas to run deterministically and to process requests sequentially according to a total order. We argue that these constraints could impede the adoption of fault tolerance techniques in practice, for example:

- Practical systems often involve nondeterministic operations when they execute clients' requests, and states of the replicas could diverge if the application nondeterminism is not controlled.

- Executing requests at the replicated server sequentially according to a total order often results in unacceptable low system throughput and high end-to-end latency.

To overcome these issues, the application semantics must be considered, as demonstrated by a large number of fault tolerance systems (Burrows, 2006; Castro and Liskov, 2002; Chandra et al., 2007; Cowling et al., 2006; Kotla et al., 2009; Li et al., 2012; MacCormick et al., 2004; Moraru et al., 2013; Zhao, 2009; Zhao et al., 2009). For example, most of them have focused on building fault tolerant storage systems such as file systems and database systems, where a lighter weight mechanism is designed to handle read-only requests while update requests are totally ordered. The identification of read-only requests requires the knowledge of the application semantics of a particular application. In some systems, such as a networked file system (NFS), some requests would trigger the access of the local physical clock, which constitutes a nondeterministic operation. In (Castro and Liskov, 2002), the application semantics of NFS was used to identify this nondeterministic operation and to design a control mechanism accordingly.

The need for incorporating application semantics is further showcased by recent works on fault tolerance systems designed to operate in wide-area 
networks (Li et al., 2012; Moraru et al., 2013). In (Li et al., 2012), a hybrid replica consistency model is used to reduce the end-to-end latency and enhance the system throughput for wide-area fault tolerance systems based on state machine replication. In this hybrid model, commutative requests (referred to as Blue operations) are executed locally and the operations are asynchronously disseminated to other replicas using an eventual consistency model. On the other hand, a strong consistency model is used for requests that have inter-dependencies (referred to as Red operations). Furthermore, some operations that are not directly commutative are converted into commutative operations (the converted operations are referred to as shadow operations) for even better performance. The determination on whether or not two requests are commutative, and the transformation of some pairs of requests into commutative requests, all require the knowledge of application semantics.

In (Moraru et al., 2013), a new variation of the Paxos consensus algorithm, referred to as Egalitarian Paxos (or EPaxos), was proposed for state machine replication. EPaxos requires every request to indicate whether or not it is read-only, and to provide the set of requests on which it depends for each update request. Total ordering is needed for conflicting requests only and non-conflicting requests are executed concurrently, thereby, significantly increasing the system throughput. It is apparent that application semantics is essential for EPaxos to work properly.

We start this review by elaborating why considering application semantics for state machine replication is necessary. We then propose a classification of various approaches to building practical fault tolerance systems. This is followed by the description of the actual performance engineering mechanisms under the classification framework. We conclude this article by outlining potential future research in high performance fault tolerance computing.

This article is based on our previous work in attempting to classify existing approaches to designing practical Byzantine fault tolerance systems by incorporating application semantics. We are not aware of other work that aims to provide a systematic review of this subject. In (Zhao, 2014b), we coined the term "application-aware Byzantine fault tolerance" to refer to this line of work, and compiled known research works roughly based on their complexity. In (Zhao, 2014a), we proposed our first classification framework. In this article, we have expanded the classification to include both conservative Byzantine fault tolerance and optimistic Byzantine fault tolerance. Furthermore, we have widened the scope of the classification to include state 
machine replication with both the crash fault and Byzantine fault models.

The ultimate goal of our research is to provide a guideline on designing high performance practical fault tolerance systems by identifying when a distributed agreement is needed, and on what operations. This is analogous to the challenge of building a secure system, where one must know when and where to use security primitives, such as encryption and digital signatures. We believe that it is time to treat distributed agreement algorithms as basic building blocks for dependable systems the same way as security primitives to secure systems. The use of security primitives alone does not warrant a secure system. Similarly, the use of distributed agreement in a naive manner does not necessarily enhance the dependability of a system. It is essential to know exactly when to use the tool and on what operations. This cannot be accomplished without considering application semantics.

The contributions of this review include:

- We present a strong argument that it is not practical to treat the sever application as a black box when replicating it for fault tolerance and, that one cannot ignore the application semantics in dependability design. In addition to performance overhead and replica consistency issues, we identify scenarios when the replicated system may deadlock if requests are executed sequentially.

- We propose a classification framework for various performance engineering approaches to fault tolerance, together with the description of the mechanisms used in these approaches. They could serve as a guideline on designing practical dependable systems.

- We outline potential future research directions that could reduce the cost of application semantics discovery and the maintenance of custom fault tolerance implementations.

\section{Why Application Semantics Matters}

Fault tolerance algorithms designed for state machine replication concern only the total ordering of requests to be delivered to the replicated server replicas. Hence, such algorithms can be used by any application as long as the replicated component acts deterministically as a state machine, i.e., given the same request delivered in the same total order, all replicas would go through the same state transitions (if any), and produce exactly the same 
reply. However, this does not mean that we should treat each application as a black box and employ a fault tolerance algorithm as it is by totally ordering all requests and executing them sequentially according to the total order. In the following, we present three major motivations for exploiting application semantics in fault tolerance.

\subsection{Reduce Runtime Overhead}

There are two main types of runtime overhead introduced by state machine replication based fault tolerance algorithms:

1. Communication and processing delays for each remote invocation due to the need for total ordering of requests, which would impact the endto-end latency.

2. The loss of concurrency degrees at the replicated server due to the sequential execution of requests, which impacts the system throughput (i.e., how many requests can be handled by the replicated server per unit of time).

By exploiting application semantics, we can introduce the following optimizations:

- Reducing the end-to-end latency by not totally ordering all requests. There is no need to totally order read-only requests (i.e., requests that do not modify the server state). Additionally, for some stateless session-oriented applications, source ordering of requests may be sufficient (Chai and Zhao, 2013).

- Enabling concurrent processing at the server replicas for some requests. Non-conflicting requests can be executed concurrently (Kotla and Dahlin, 2004; Raykov et al., 2011). Doing source ordering alone for requests also increases the system throughput (Chai and Zhao, 2013).

- Furthermore, deferred agreement for session-oriented applications (using one distributed agreement instance for a group of requests typically at the end of the session), could further increase the system throughput (Zhang et al., 2012). 


\subsection{Respect Causality and Avoid Deadlocks}

General purpose fault tolerance algorithms are designed for simple clientserver applications where clients do not directly interact with each other and send requests to the replicated server independently. For multi-tiered applications with sophisticated interaction patterns (Chai and Zhao, 2012b), the basic assumption for these fault tolerance algorithms may no longer hold and hence, if used in a straightforward manner, may lead to two problems: (1) causality violation, when the total order imposed on two or more requests is different from their causal order and, (2) deadlocks, when sequential execution of requests is imposed when concurrent processing of some requests is mandatory according to the application design (Zhao et al., 2005a). In these cases, application semantics must be tapped to discover the causal ordering between requests and identify what requests must be delivered concurrently. Otherwise, the integrity and the availability of the system could be lost.

\subsection{Control Replica Nondeterminism}

State machine replication requires that replicas behave deterministically when processing requests. However, many applications are involved with some form of nondeterministic operations, such as taking a timestamp and generating a random number using a pseudo random number generator. Without considering the application semantics, it is usually not possible to know whether or not nondeterministic operations would be involved in request processing (Zhang et al., 2011). Additionally, multithreaded applications are pervasive and it is particularly difficult to control the nondeterminism caused by multithreading. In fact, it is a huge hindrance for applications to use state machine replication.

If such nondeterministic operation is not controlled, the states of the replicas might diverge and the replicas might produce different replies to the client. Hence, exploiting application semantics not only helps in minimizing the runtime overhead, but also helps guarantee the safety and integrity of fault tolerance systems (especially for Byzantine fault tolerance systems).

\section{Classification of Performance Engineering Approaches}

As shown in Figure 1, performance engineering approaches for state machine replication can be divided into two different camps. In one camp, approaches are developed to achieve conservative fault tolerance. In the

other camp, the performance improvement is accomplished by executing a 
request before the total order of the request has been established, hence, the name optimistic fault tolerance. In conservative fault tolerance, the focus is to ensure the consistency of message execution order at all nonfaulty replicas. The objective of optimistic fault tolerance, on the other hand, is to speculatively execute requests as early as possible and execute multiple requests concurrently whenever possible. As a tradeoff, nonfaulty replicas may become inconsistent temporarily, hence, appropriate mechanisms must be designed to ensure prompt detection of, and recovery from, inconsistency of the replicas. Compared with conservative approaches, optimistic fault tolerance protocols offer better performance at the cost of poorly masking faults, i.e., they can more easily affect liveness properties and render the system unusable.

\subsection{Conservative Fault Tolerance}

Within the camp of conservative fault tolerance, we can further divide the approaches based on two different criteria. The first criterion concerns the types of operations performed at each replica. The second criterion concerns whether or not the context to which the request belongs is considered.

According to the first criterion, we classify the approaches based on whether or not the operations on state objects are limited to basic or complex types.

- Basic operation: An operation is basic if it is a read or write operation on a state object, or the creation or deletion of an object. Furthermore, if the operation is write or create, the value of the object is set deterministically, i.e., it is either provided by the client that issues the request, or set by the system deterministically. Note that the create operation may effectively access two state objects. When an object factory is used, or the object is created within a specific hierarchy such as within a particular directory, the create operation will inevitably access both the object factory or the parent directory, and the object created.

- Complex operation: it refers to an operation beyond basic operations, such as increment and decrement for integer numbers, and append or truncate for text strings. We also consider an operation complex if it sets a new value to a state object nondeterministically, such as using the local clock value or using a value produced by a pseudo random number generator. 

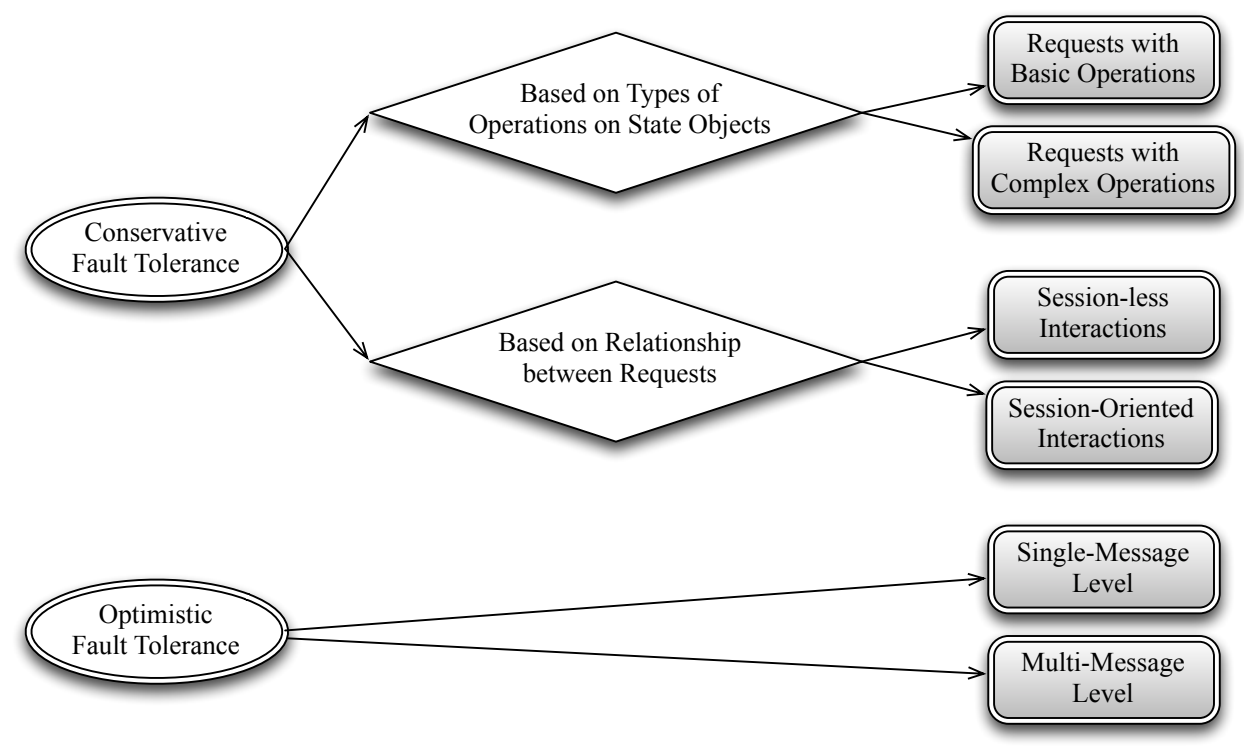

Figure 1: Classification of various approaches to performance optimization for state machine replication.

According to the second criterion, if the context is not considered (or there is no such context), requests from different clients are assumed to be sent independently. On the other hand, if a context is present, it correlates a number of requests issued by the same or different clients within the same session. If a request is issued within such a session, the representation of the context is typically included in the message.

We first discuss the resulting two categories using the first criterion. We then elaborate the implications of the context as used in the second criterion.

\subsubsection{Requests with Basic Operations}

For this category, the relationship between different requests is determined by the target state objects and the corresponding operations. Two requests are considered inter-dependent if they access at least one common state object and the operation on that object from any of the requests is a write operation. Otherwise, requests are considered independent.

Note that for a write or a create operation, we assume that the new value is determined deterministically. Furthermore, different create operations are independent. Similarly, different delete operations are independent as well. However, create and delete operations must be totally ordered with respect 
to each other (i.e., they are considered inter-dependent).

For requests that are inter-dependent, they must be delivered and executed sequentially according to some total order at all nonfaulty replicas to ensure strong replica consistency. Independent requests, on the other hand, can be delivered and executed concurrently with respect to each other.

\subsubsection{Requests with Complex Operations}

For this category, a request may be involved with basic as well as complex operations. The dependency among the requests can be analyzed in a similar manner as that for requests with basic operations by classifying complex operations into read-oriented and write-oriented types. However, such analysis is not adequate.

Even if two write-oriented requests access the same state object, they might not be conflicting with each other depending on the complex operations involved. For example, if both requests are involved with the increment operation on the same state object, they are considered commutative and hence, can be delivered and processed concurrently.

The complexity of the operation could also require additional mechanisms to ensure strong replica consistency. For example, if either the value to be returned to the client, or the new value to be assigned to the state object is determined based on the reading of the local clock, or on the output from a pseudo random number generator, additional mechanisms are required to make sure that the same value is chosen by all nonfaulty replicas (Zhao, 2008).

\subsubsection{Session-Oriented Interactions}

For session-less interactions between clients and server replicas, requests are assumed to be sent independently. Hence, server replicas may determine a total order of the requests sent by different clients arbitrarily (usually according to the order in which they are received at the primary). For concurrent requests, they may be batched together for total ordering. Obviously, requests issued by the same client are ordered according to the order in which they are sent to preserve causality.

For requests sent within a session, however, they may be correlated. Furthermore, session-oriented applications typically are multi-tiered. This correlation among the requests imposes the following constraints on the ordering and handling of requests: (1) the total order of requests must respect their causality, and (2) some nested invocations may have to be executed before 
another request is fully processed. Issues related to these constraints and the corresponding solutions will be presented in Section 5.3.1 and Section 5.3.2. On the other hand, the correlation among the requests within a session also offers additional opportunities for optimization such as source ordering (Chai and Zhao, 2013) and deferred agreement (Zhang et al., 2012), which will be elaborated in Section 5.3.3 and Section 5.3.4.

\subsection{Optimistic Fault Tolerance}

Optimistic fault tolerance under the crash fault model has been well studied (Li et al., 2012; Moraru et al., 2013; Saito and Shapiro, 2005). The first form of optimistic Byzantine fault tolerance is referred to as tentative execution (Castro and Liskov, 2002) or speculative execution (Kotla et al., 2009). This form of optimization allows a replica to execute a request before knowing that the request has been totally ordered, and yet the requests are still executed sequentially at each replica. Hence, we call this form of optimization single-message optimistic fault tolerance. The potential benefit of this form of optimistic fault tolerance is the reduced end-to-end latency perceived by clients.

In (Chai and Zhao, 2014a,c,b; Zhao and Babi, 2013), a more aggressive form of optimistic fault tolerance approach is employed where replicas execute multiple requests concurrently and synchronize their state periodically or ondemand when an inconsistency among the replicas has been detected. We call this approach multi-message optimistic fault tolerance. In this approach, the end-to-end latency can be reduced and the throughput may be increased significantly. As a tradeoff, optimistic multi-message fault tolerance ensures a weaker form of consistency called eventual replica consistency (Saito and Shapiro, 2005).

Note that the nature of some applications dictates that multi-message optimistic fault tolerance be used, such as realtime collaborative editing applications where multiple users expect to be able to update the shared document concurrently (Sun et al., 1998). Optimistic fault tolerance would also be required when the availability outweighs the consistency of the system (Brewer, 2012; Ramakrishnan, 2012; Saito and Shapiro, 2005).

\section{System Model}

The asynchronous distributed system model is assumed when establishing the safety property of all performance engineering mechanisms described in 
Section 5 and Section 6 . The liveness property is ensured only when the system is behaving partially synchronously.

For client-server applications, the server is replicated with sufficient replication degrees. For multi-tiered applications, the middle-tier servers or the backend server is replicated in the same way as the client-server applications except that a replica may issue nested requests to, and receive the corresponding replies from, other components in the system.

For state machine replication with the crash fault model, we assume that $n=2 f+1$ replicas are used to tolerate up to $f$ faulty replicas. For state machine replication with the Byzantine fault model, we assume that $n=$ $3 f+1$ replicas are used to tolerate up to $f$ faulty replicas. $3 f+1$ is the optimal number of replicas for the Byzantine fault model (Castro and Liskov, 2002) as explained in the following. A replica has to make a decision when it has collected inputs from $n-f$ replicas because up to $f$ replicas might be faulty. Among the $n-f$ inputs, up to $f$ of them might have come from faulty replicas. Hence, only $n-2 f$ of them may be sent from nonfaulty replicas. We want to ensure that the majority of inputs are from nonfaulty replicas. Hence, $n-2 f$ must be greater than $f$, which means that $n>3 f$. Therefore, the smallest total number of replicas needed for a system to tolerate up to $f$ faulty replicas is $3 f+1$. Additionally, under the Byzantine fault model, messages exchanged must be protected by an unforgeable security token to prevent a faulty replica from impersonating as another nonfaulty replica. In most cases, a message authentication code could be used as the security token. For view-change related messages, a digital signature is typically used as the security token for stronger protection.

We assume that a replica is either stateless or stateful. A stateless replica simply process a request based on some predefined application logic and it does not maintain any state for any client across different replicas. A stateful replica consists of one or more state objects, and the processing of a request may result in the change of one or more state objects.

Furthermore, we assume that the application is designed to execute concurrent requests with multiple threads, such as in a thread pool, for maximum throughput as well as for the capability of handling multiple concurrent clients. It is not interesting to consider applications that use the singlethreaded execution model in this review, because such applications fit the state machine replication requirement well naturally.

Unless otherwise stated, we assume that replicas are directly involved with distributed consensus to ensure the agreement of the message total ordering 
and other values if necessary, although the task of distributed agreement (Byzantine agreement in particular) can be separated out and provided as a service by a separate cluster (Clement et al., 2009; Yin et al., 2003; Chai and Zhao, 2012a).

\section{Conservative Fault Tolerance Mechanisms}

In this section, we describe the mechanisms for conservative fault tolerance in three parts. In the first two parts, we elaborate the mechanisms designed for basic operations, and those to accommodate complex operations, respectively. These mechanisms are applicable to both session-less and session-oriented applications. In the third part, we present the mechanisms designed specifically for session-oriented application, regardless of the type of operations (i.e., basic or complex).

\subsection{Requests with Basic Operations}

Research on requests with basic operations is heavily influenced by techniques developed for transaction processing (Gray and Reuter, 1992), in particular, the concurrency control theories. This is not surprising because the problem layout for requests with basic operations is rather similar to that for transaction processing. It is intuitive to identify and handle read-only requests differently from requests that modify the server state so that the end-to-end latency for read-only requests is minimized. Requests that might modify one or more state objects are analyzed for potential dependency based on the type of operations (e.g., read or write) and target state objects. In a conservative approach introduced in Section 5.1.2, a partial order is imposed on the requests that allows independent requests to be processed in parallel. A special case is requests partitioning, where requests that operate on disjoint state objects can be separated based on some simple measure and handled concurrently without further dependency analysis.

\subsubsection{Read-Only Requests}

It is well-known that the majority of operations are read-only. Hence, it has long been a design principle to optimize for read-only operations. This is the case for replicated database systems (Gray and Reuter, 1992), group communication systems (Birman, 1985), and state machine replication with the crash fault model in the pre-Paxos era (Felber, 2001). Interestingly, it is not straightforward to optimize read-only operations for state machine 
replication based on the classic Paxos algorithm as pointed out in (Chandra et al., 2007). By default, read-only requests are totally ordered with respect to all other requests in Paxos to ensure that the most up-to-date state is returned. One may attempt to allow the read from the primary replica alone without going through an instance of Paxos. However, doing so may risk of reading stale data if another replica has been elected to be the new primary and the previous primary is not aware of the situation. In (Chandra et al., 2007), a master lease mechanism (Gray and Cheriton, 1989) is used to ensure that no other replica could be promoted to the primary role while the current primary is still holding the release. The master lease mechanism enables the primary to serve read-only requests directly without having to run an instance of the Paxos.

The first mechanism designed for read-only requests in the context of Byzantine fault tolerance is described in (Castro and Liskov, 2002). A readonly request can be delivered at a replica immediately without being totally ordered first. To ensure that it reads from the updates that are causally preceding the read, the client collects $2 f+1$ matching reply messages before it delivers the reply. In case when the client fails to collect $2 f+1$ matching replies, it resends the request as a non-read-only request. Note that here we assume that the total number of replicas $n$ is $3 f+1$. If $n>3 f+1$, the number of matching reply messages would increase accordingly (Malkhi and Reiter, 1998).

\subsubsection{Concurrent Execution of Independent Requests}

The first mechanism to enable concurrent execution of requests in the context of Byzantine fault tolerance is introduced in (Kotla and Dahlin, 2004). The center piece of the mechanism is a predefined concurrency matrix that can be used to determine whether or not two requests are independent based on the operation and argument (representing the state object to be accessed) specified in the request. The message delivery is controlled by a parallelizer component that is sandwiched between the Byzantine agreement layer (for message total ordering) and the server application (referred to as executor in (Kotla and Dahlin, 2004)), as shown in Figure 2.

The parallelizer dynamically tracks the dependency of requests that have been totally ordered according to the concurrency matrix. The concurrency matrix is populated based on application-specific rules. Furthermore, it is assumed that a particular thread model is used where threads from a threadpool ask the parallelizer for the next requests to execute. 


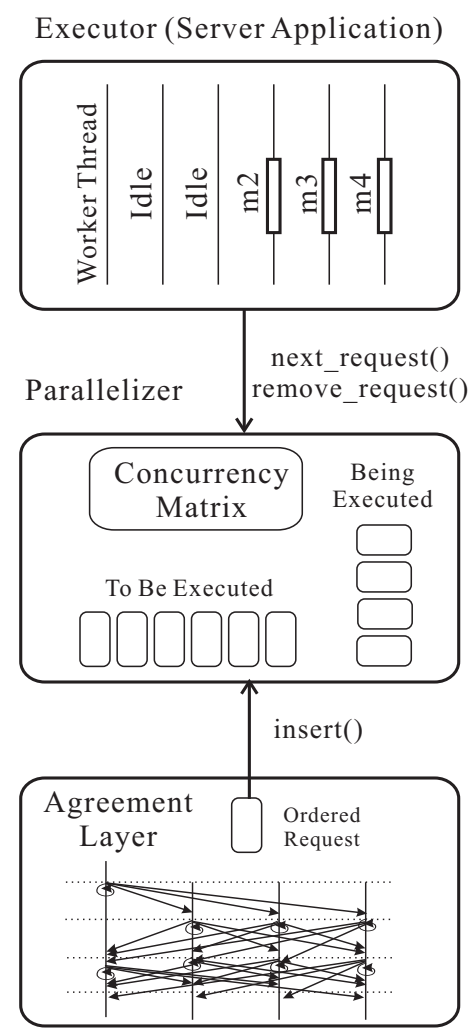

Figure 2: The architecture of the parallelizer framework for concurrent execution of independent requests.

The method insert() provided by the parallelizer is called when a request has been totally ordered. The inserted request is then added to the "tobe-executed" queue. The next_request() method is called when a worker thread wants to retrieve the next request to execute. The remove_request () method is invoked when a worker thread completes the execution of a request. When remove_request() is called, the request is removed from the "beingexecuted" queue.

When the next_request( $)$ method is called, the parallelizer first checks if the "to-be-executed" queue is empty. If true, the parallelizer blocks the call. Otherwise, the parallelizer decides on which request to return according to the following rules:

- If no message is in the "being-executed" queue, the first request in the "to-be-executed" queue is returned. 
- If there is one or more messages in the "being-executed" queue, the first request in the "to-be-executed" queue is examined based on the concurrency matrix to determine if there exists any requests in the "being-executed" queue that conflict with this request. The message is returned only if it does not conflict with any request in the "beingexecuted" queue.

Requests partitioning can be considered as a special form of concurrent execution of independent requests. This is because requests that belong to different partitions are naturally independent, hence, they can be executed concurrently. Requests can be partitioned if they operate on disjoint state objects. Typically, requests can be separated based on some simple measure, such as an identifier contained in the request, without complicated dependency analysis. Requests that belong to different partitions can be executed concurrently and may be handled by different groups of server replicas for scalability. This approach is seen to be employed in farsite (Adya et al., 2002), as well as in Byzantine fault tolerant Web services coordination (Chai et al., 2013; Zhang et al., 2012).

In farsite (Adya et al., 2002), requests partitioning is used to enhance the scalability of a Byzantine fault tolerant storage service. The basis for the requests partitioning is the pathname contained in the client's requests. Based on the pathname, a request is forwarded to the designated group of replicas for processing. To minimize the load on the root directory group (hence, to facilitate scalability), each client caches the mapping of the pathnames and their corresponding directory groups. When sending a request, the client identifies the target directory group by finding the longest-matching prefix in the cached mappings.

In Web services coordination (Chai et al., 2013; Zhang et al., 2012), it was recognized that requests for different atomic transactions or business activities are processed by different coordinator objects. Hence, requests arriving at the replicated coordination service can be partitioned based on atomic transactions or business activities.

\subsubsection{Transaction Processing}

The concurrency degree of requests execution can be further enhanced by treating the operations for each request as part of an atomic transaction (typically via software transactional memory (Shavit and Touitou, 1995)) and employing some concurrency control mechanism developed for transac- 
tion processing systems (Gray and Reuter, 1992). This is because in this approach, non-conflicting operations may be executed in parallel instead of sequentially for inter-dependent requests. In essence, the granularity of concurrency control is reduced from the request level (with each request consisting of several operations on a number of state objects) to the state object level.

To ensure strong replica consistency, a concurrency control mechanism must produce a serializable schedule for inter-dependent requests according to some total order at all nonfaulty replicas. The timestamp-based concurrency control may be adapted for this purpose by using the sequence number as the timestamp. Because a request is totally ordered at all nonfaulty replicas, they see the same sequence number for the same request, guaranteeing identical serializable schedule for all transactions (mapped from the requests) at all nonfaulty replicas. This approach has been used to enable concurrent Byzantine fault tolerance (Zhang and Zhao, 2012) based on a lock-free software transactional memory library (Brito et al., 2009).

Note that the transaction processing model described here and in Section 5.3.4 deviates from the traditional state machine replication technique. Nevertheless, both rely on distributed consensus or reliable totally ordered multicast to ensure replica consistency.

\subsection{Requests with Complex Operations}

In addition to the mechanisms designed for handling requests with basic operations, current research has focused on the following two aspects of complex operations: (1) commutative requests and (2) replica nondeterminism.

\subsubsection{Commutative Requests}

Even if two requests access the same object and at least one of them engages in a write-oriented operation, they might not be conflicting with each other depending on the complex operations involved. For example, if both requests are involved with the increment operation on the same state object, they are considered commutative and hence, can be delivered and processed concurrently. Apparently, requests that update different state objects are naturally commutative as well. Hence, all independent requests as defined in Section 5.1.2 are commutative, i.e., commutative requests form a superset of independent requests.

If some requests are commutative while others are conflicting with each other, the mechanism described in Section 5.1 .2 can be easily modified to 
accommodate commutative requests. More specifically, the entries corresponding to commutative requests will be labeled as non-conflicting. No additional changes are required.

One way to systematically ensure that all requests to a service are commutative is to implement the service using commutative (or convergent) replicated data types (Shapiro et al., 2011). In this case, it is possible to synchronize the state of the replicas only when it is necessary or periodically (Chai and Zhao, 2014a). We refer to this approach as multi-message optimistic fault tolerance, which is reviewed in Section 6.2.

Note that the difference between independent requests and commutative requests lies in the technique in making the determinations of these requests. If one makes a dependency check between two requests only by examining whether or not they access the same state object, then only independent requests can be identified. More sophisticated mechanisms, which inevitably would involve with application semantics, are needed to identify commutative requests that update the same state object(s). Additionally, it is worth noting that some non-commutative operations can be rendered commutative by using shadow operations (Li et al., 2012), and by operational transformation (Ellis and Gibbs, 1989).

\subsubsection{Replica Nondeterminism}

Some simple location-specific nondeterminism can be masked by using a wrapper function. The wrapper translates location-specific values such as process id to a group-wide id, which would ensure strong replica consistency. This practice has long been used to build fault tolerance systems (Zhao et al., 2013) and is used in (Castro et al., 2003) as an extension to PBFT. The use of a wrapper does not introduce any additional communication step.

In (Zhang et al., 2011), a classification of replica nondeterminism beyond simple wrappable types is provided and a set of mechanisms for controlling replica nondeterminism is presented. As shown in Figure 3, three criteria are used to classify different approaches: the wrapability of nondeterministic operations, the determinability of nondeterministic operations and their associated values before a request is executed, and the verifiability of nondeterministic values proposed by a replica.

- Wrapable nondeterminism: The nondeterministic values of this type at different replicas can be made consistent using a predefined wrapper function. The wrapper function uses the group identity together with a 


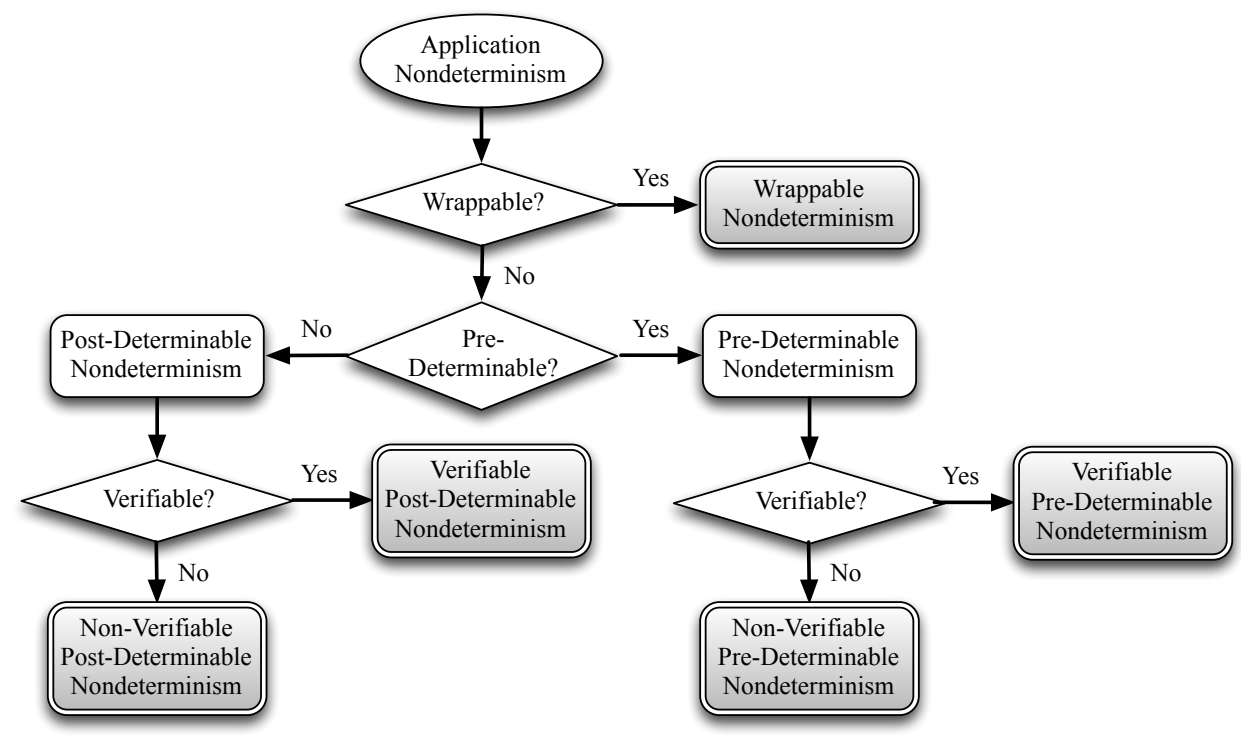

Figure 3: A classification of nondeterminism types.

deterministic local algorithm to compute various local identifiers (such as process ids and file descriptors) that would otherwise be different at different replicas (Castro et al., 2003).

- Pre-determinable nondeterminism: Although the nondeterministic variables of this type can be determined before a request is executed, the nature of the operations dictates that they cannot be set according to some predefined deterministic algorithm, such as the use of random numbers. Usually, such values should be determined collectively by replicas.

- Post-determinable nondeterminism: The nondeterministic values of this type cannot be determined before a request is executed. Hence, such values would have to be initially recorded during the execution of a request at the primary, and the values will then be propagated to backup replicas for their verification and adoption. For example, it is virtually impossible to predefine the thread interleaving for a multi-threaded application prior to the execution of a request. The only practical way is to record such interleaving at the primary and enforce the same interleaving at the backups. 
The last two types can additionally be divided according to the verifiability of the values proposed by a replica:

- Verifiable nondeterminism: The nondeterministic values proposed by a replica can be easily verified.

- Nonverifiable nondeterminism: The nondeterministic values proposed by a replica cannot be easily verified. For instance, it is difficult to verify a random number proposed by a replica. In this case, the final values to be adopted for each request would have to be collectively determined by the majority of nonfaulty replicas. This makes it impossible for a faulty replica to dictate a wrong value for the group of replicas.

In summary, as shown in Figure 3, there are five types of application nondeterminism: wrapable nondeterminism, verifiable predeterminable nondeterminism, nonverifiable pre-determinable nondeterminism, verifiable postdeterminable nondeterminism, and nonverifiable post-determinable nondeterminism.

Some of the nondeterminism can be controlled by adding one more communication step to the Byzantine agreement step for the request to guarantee that the same nondeterministic decision are made by nonfaulty replicas. Other nondeterministic decisions must be controlled via an extra Byzantine agreement instance. For detailed description of the mechanisms, readers are referred to read (Zhang et al., 2011).

\subsection{Session-Oriented Interactions}

A unique aspect of session-oriented interactions is the correlation of requests that belong to the same session. In this section, we explain the two potential issues caused by the correlation of requests as we identified in Section 3.1.3 in detail, and describe the corresponding mechanisms to overcome them. We also elaborate how to improve the system performance by exploiting the correlation among requests with a session.

\subsubsection{Preventing Causality Violation}

The most obvious constraint due to requests correlation is that the replicas can no longer impose a total order according to the order in which the requests are received because doing so might violate the causal ordering among the requests. Consider the example scenario shown in Figure 4. If process A sends a request $m_{1}$ to server replicas and subsequently issues a request $m_{2}$ 


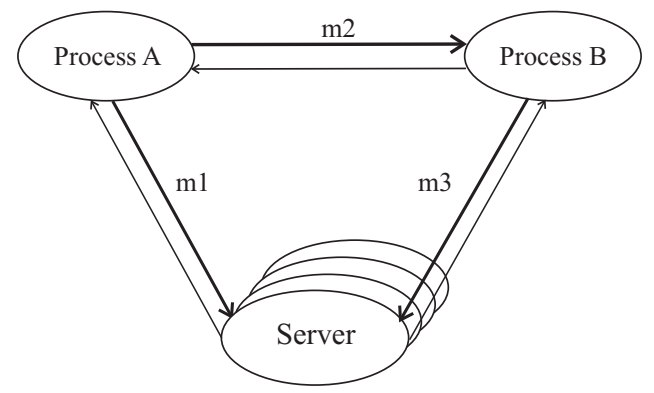

Figure 4: A scenario that two requests might be causally related. In this example, $m_{3}$ might causally depends on $m_{1}$, hence, $m_{3}$ must be ordered and delivered after $m_{1}$ has been processed.

to process $\mathrm{B}$ (i.e., A sends $m_{2}$ prior to receiving the reply for $m_{1}$ ). Subsequently, process $\mathrm{B}$ issues a request $m_{3}$ to the server replicas. It is apparent that $m_{1}$ must be ordered ahead of $m_{3}$ because $m_{3}$ might depend on $m_{1}$.

The causality violation issue might arise only when clients communicate directly with each other without the knowledge of the replicated server, as shown in Figure 4. In multi-tired session-oriented applications, this may happen frequently. For example, in a Web services atomic transaction or business activity, participants often communicate with each other as well as with the coordination service (Chai et al., 2013; Zhang et al., 2012). In these applications, the causality of requests is often reinforced at the application level, e.g., a request is queued until the one that precedes it is received (Chai et al., 2013; Zhang et al., 2012). Therefore, for applications that have provisions for causality detection and enforcement, the causality violation issue goes away if we allow concurrent delivery of requests.

\subsubsection{Avoiding Deadlocks}

In multi-tiered applications, nested remote invocations are common. If two or more replicated servers issue nested invocations to each other in response to requests sent from their clients, the nested requests must be delivered and executed prior to the initial requests are fully processed. Dictating a sequential order in the execution of the requests from the clients and those for nested invocations could lead to deadlocks. To see why, consider the scenario shown in Figure 5 where two clients (Client 1 and Client 2) concurrently issue requests to two correlated replicated services (Service A and Service B), respectively. As the result of the remote invocation UpdateA() at Service A, a nested invocation $\operatorname{NestedB(})$ is issued by Service A on Ser- 

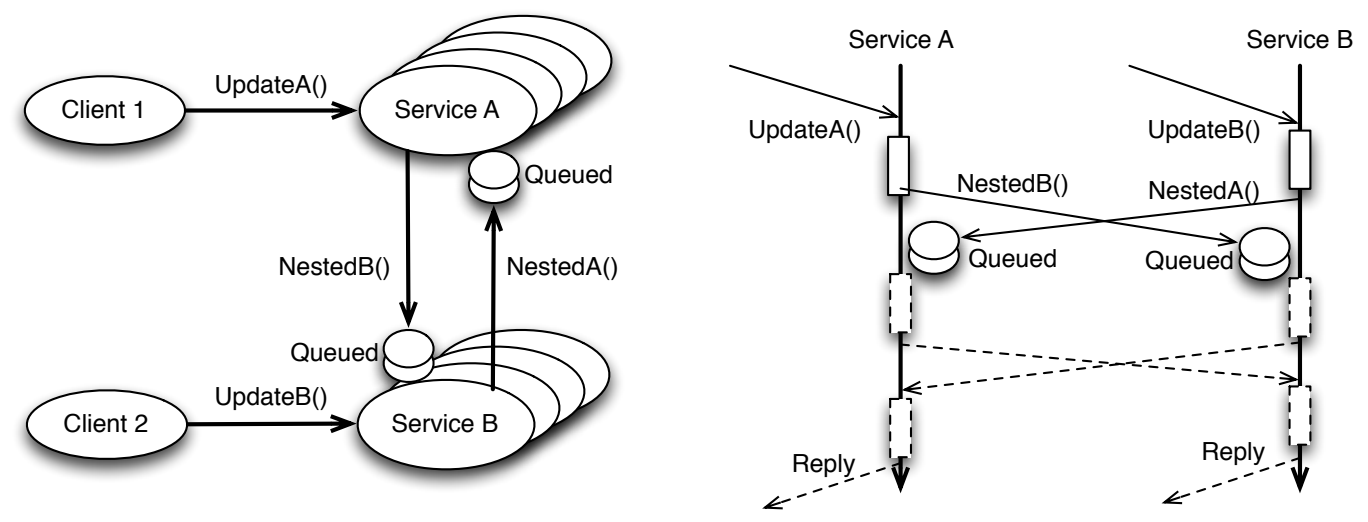

Figure 5: A scenario that could lead to deadlock without concurrent processing of requests to the replicated servers. The sequence diagram on the right additionally illustrates how to break the deadlock using steps represented in dotted lines.

vice B. Similarly, a nested invocation NestedA() is issued by Service B on Service A as the result of the invocation from Client 1. According to the state machine replication requirement, all requests on a replica must be serialized, i.e., NestedA() must be delivered and executed after UpdateA() at Service A, and NestedB() must be delivered and executed after UpdateB() at Service B. Hence, NestedA() and NestedB() will be queued. However, doing so would prevent Service A from completing the invocation UpdateA() from Client 1 because it blocks waiting for the reply for its nested invocation NestedB() on Service B, and similarly, this would prevent Service B from completing the invocation UpdateB() from Client 1.

In principle, the deadlock problem can be resolved with concurrent delivery of requests that have causal relationship, as illustrated on the right side of Figure 5 using dotted lines. This inevitably violates the basic requirement of state machine replication, and it is not trivial to allow concurrent invocations without compromising the replica consistency and without inter-replica communication. A mechanism was introduced in (Zhao et al., 2005a) to accomplish this task, which requires the interception of all mutex acquisition and release operations and the use of a set of data structures to keep track of the concurrency control policy, messages sent and received, and active and blocked threads.

The main challenge is to identify exactly when the next request could be delivered. The complexity of this problem is due to: 


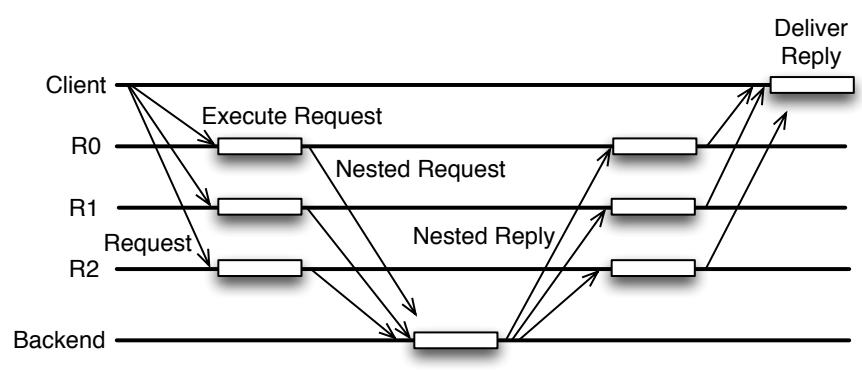

Figure 6: Normal operation of source-order based protocol.

- A replica might receive more than one message to be handled by the same thread.

- A thread might hold on to a mutex until a sequence of nested invocations have been completed.

In either case, it is not safe to deliver a new request to the replica even if all threads are blocked because in the first case, some of the threads might be waiting for a nested reply, and in the second case, uncontrolled concurrent multithreading might occur, which could induce replica inconsistency.

To deal with the first case, a new request can be delivered only when all threads in the replica have become quiescent, i.e., all requests have been delivered and no thread is holding a mutex. The mechanism to handle the second case is much more complicated because a new request must be delivered to a replica when it is not quiesced. The basic idea is to make sure that only one thread is active at a time. For details, readers are referred to (Zhao et al., 2005a).

\subsubsection{Source Ordering}

For some multi-tiered applications with a replicated middle-tier server and a backend server managing all persistent state, it is argued in (Chai and Zhao, 2013) that maintaining source ordering of requests is sufficient either due to the commutativity of the operations at the replicated middle-tier server, or due to the constraint imposed by the database server. Furthermore, only $2 f+1$ middle-tier server replicas are required when up to $f$ of them could be Byzantine faulty. A client, as well as the backend server, must collect $f+1$ matching reply messages or nested request messages to deliver the reply or request, as shown in Figure 6. 


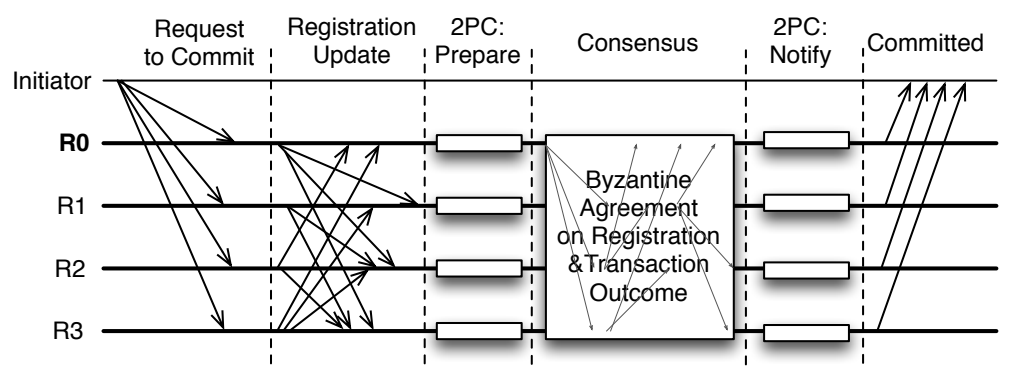

Figure 7: Deferred agreement mechanism in the context of a distributed transaction.

\subsubsection{Deferred Agreement}

A session by definition defines the beginning and the ending of an interaction between a group of participants. For some requests received at the replicated server, their total order can be determined right before the end of the session, thereby, reducing the runtime overhead. Deferred agreement differs from the batching mechanism used in general purpose fault tolerance systems (Moser et al., 1996; Castro and Liskov, 2002) because batching is done to a group of requests that arrive concurrently, while deferred agreement applies to requests received over time during a session.

Many session-oriented applications adopt the transaction processing model where a group of operations within the session (i.e., the transaction) are executed atomically (i.e., either they are all successful or as if none of them has been executed). In this case, some of the operations can be delivered immediately during the session, and a sanity check on potential conflicts can be performed at the end of the session, which constitutes a deferred agreement on the group of operations within the session. Deferred agreement also applies to distributed transactions where the two phase commit (2PC) protocol is used.

In the context of deferred agreement, the total ordering of individual requests is no longer important. What has to be agreed upon by all nonfaulty replicas is the collection of requests that have been deferred. For a distributed transaction, the votes collected in the first phase of the two-phase commit and the corresponding transaction outcome should also be agreed upon by nonfaulty replicas. The agreement of all such information can be accomplished by using a single instance of distributed agreement, as shown in Figure 7.

For the deferred agreement mechanism to work, a transaction partici- 
pant must ensure that its registration request has reached at least $2 f+1$ out of $3 f+1$ transaction coordinator replicas (Zhang et al., 2012). When a replica receives a registration request, it adds the participant to its registration record. When the initiator requests the coordinator to commit a distributed transaction, an extra phase of message exchange is carried out prior to the start of $2 \mathrm{PC}$. In this phase, which is referred to as the registration update phase, each coordinator replica broadcasts its registration record to all other replicas. This phase ends at a replica when it has collected $2 f$ update messages from other replicas. The objective of this phase is to ensure that every nonfaulty replica has the registration records of all nonfaulty

participants. At the end of this phase, each replica computes a superset of registration records.

At the conclusion of the first phase of $2 \mathrm{PC}$, a coordinator replica determines the outcome of the transaction (i.e., commit or rollback). The decision certificate is used as the evidence for every transaction outcome proposed by a replica. The decision certificate is a critical data structure that contains the registrations and the votes from participants collected in the first phase of $2 \mathrm{PC}$. According to $2 \mathrm{PC}$, a transaction can be committed only when all participants have voted to commit the transaction in the first phase of $2 \mathrm{PC}$. Hence, the registration records matter because a faulty replica could try to abort a transaction when it should not by excluding a participant from the membership of the transaction.

In the ensuing consensus phase, replicas build an agreement on both the decision certificate and the outcome of the transaction. When an agreement is reached, a replica notifies all participants about the transaction outcome, and then informs the initiator about the outcome of the transaction.

\section{Optimistic Fault Tolerance Mechanisms}

There are two levels of approaches to optimistic fault tolerance. In a relatively conservative approach, it is assumed that replicas are rarely faulty (especially for the primary) and there usually exists a single leader. Hence, a replica tentatively accepts the ordering for a request before a full distributed consensus has been reached (for server-side speculation), and a client tentatively accepts a reply as soon as it receives the first reply from any of the replicas (for client-side speculation in a Byzantine fault tolerance system). In this approach, the speculation is done for one request at a time, hence, we refer to this approach as single-message optimistic fault tolerance. In a more 
aggressive approach, it is assumed that a very small fraction of requests are conflicting, and a replica delivers and executes a request as soon as it receives one. Naturally, a replica could have executed a number of requests before the system engages in a consistency check among replicas. Hence, we refer to this approach as multi-message optimistic fault tolerance. The former approach is often used in Byzantine fault tolerance systems because Byzantine agreement typically takes a number of communication steps. The latter approach has been used in fault tolerance systems with the crash model (Li et al., 2012; Moraru et al., 2013; Saito and Shapiro, 2005) as well as those designed to tolerate Byzantine faults (Chai and Zhao, 2014a,c,b; Zhao and Babi, 2013). In both approaches, a recovery mechanism is necessary to resolve replica inconsistency as the result of wrong speculation.

Note that single-message optimistic fault tolerance does not depend on the application semantics and hence, they can be used in any system. We include this line of research here for completeness reasons. By utilizing application semantics, more aggressive optimization can be performed, which is reviewed in Section 6.2.

\subsection{Single-Message Optimistic Fault Tolerance}

The fundamental assumption for single-message optimistic fault tolerance is that there often exists a single leader among the replicas and replicas are rarely faulty. Based on this assumption, a replica could deliver and tentatively executes a request before the total order of the message has been established in server-side speculation, and a client could tentatively accept the first reply it receives in client-side speculation.

\subsubsection{Server-Side Speculation}

The first single-message level optimistic approach for Byzantine fault tolerance was proposed in (Castro and Liskov, 2002). In this approach, a replica tentatively executes a request immediately after the message has been prepared, i.e., when $2 f+1$ replicas have accepted (or sent) a pre-prepare message for the message, and has accepted $2 f$ matching prepare messages (including the one from its own for a backup replica). Tentative execution has low risk of executing the request in a wrong order because unless the primary becomes faulty, the order for the request at the prepared stage (i.e., the prepared order) would be identical to the total order. The request might be executed in a wrong order only if the primary fails and the new primary is unaware of the prepared order for the request. The benefit of tentative execution is the 


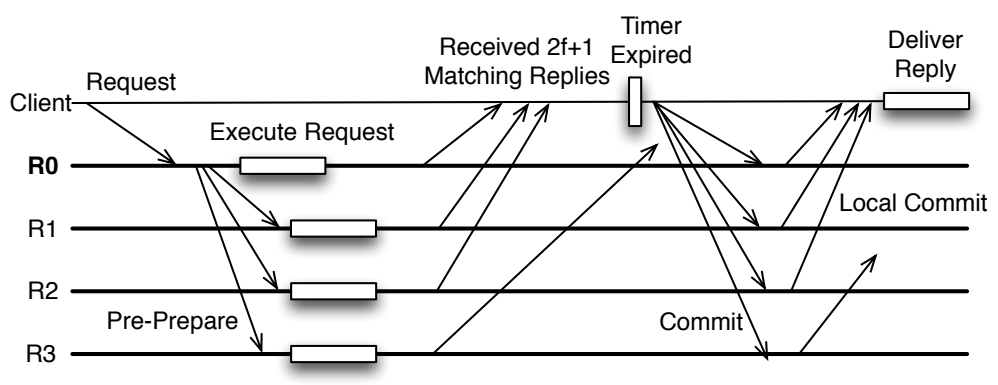

Figure 8: Speculative execution in Zyzzyva.

elimination of one communication step in the critical path of the end-to-end latency.

In speculative execution introduced in Zyzzyva (Kotla et al., 2009), a replica executes a request as soon as it has generated (for the primary) or received (for a backup) the pre-prepare message for the request as shown in Figure 8. This more aggressive approach could cut two communication steps from the end-to-end latency in the most optimal case. However, as a tradeoff, the client must wait until it has collected $3 f+1$ matching replies (i.e., from all replicas) before it accepts and delivers the reply. If the client fails to collect matching replies from all replicas, but manages to collect at least $2 f+1$ matching replies, an additional round of communication between the client and replicas becomes necessary (as shown in Figure 8). In the latter case, Zyzzyva would not have any advantage over PBFT as far as the end-to-end latency is concerned. Additionally, the view change must also be changed in Zyzzyva due to more aggressive speculation.

In both tentative execution and speculative execution, a recovery mechanism is needed to resolve the issue of wrong speculation. In PBFT (Castro and Liskov, 2002), the prepared record for a request that has been tentatively executed must be indicated explicitly so when it is disseminated in a view change. If the order as determined by the new primary is different from the order executed tentatively in the previous view, the replica must be rolled back to the latest stable checkpoint, and all logged requests ordered after the checkpoint must be re-executed, including those that have not been executed tentatively. In Zyzzyva (Kotla et al., 2009), no recovery mechanism was provided explicitly. We believe the recovery mechanism defined for tentative execution can also be used to recover from a wrong speculation. In summary, the penalty for a wrong tentative or speculative execution can be 
quite high, depending on the frequency of checkpointing.

\subsubsection{Client-Side Speculation}

In addition to server-side speculation, a client could also use speculation to reduce the end-to-end latency, as presented in (Wester et al., 2009). In client-side speculation, a client accepts and delivers the first reply received instead of doing so only when it has collected $f+1$ or more matching replies for Byzantine fault tolerance. This scheme is particularly useful in Byzantine fault tolerance systems operating in wide-area networks. To further reduce the end-to-end latency, the primary replica immediately executes a request and generates an early reply to the client as soon as it has issued a preprepare message, i.e., client-speculation is typically used in conjunction with server-side speculation.

Obviously, if the client speculation is wrong, e.g., if the first reply that the client received is sent by a faulty replica, the client must be rolled back to a previously recorded correct state. This requires periodic checkpoining at the client. A client could detect wrong speculation by continuing collecting more replies. If it could collect $f+1$ matching replies that are different from the one it has delivered speculatively, the client learns that it has speculated wrong and rolls back its state using the last checkpoint and replays the logged committed replies since that checkpoint.

Another issue is that the client might issue new requests after it has delivered a reply speculatively. Because these requests are generated based on the speculative state at the client, they might corrupt the state of server replicas, if the reply delivered speculatively turns out to be wrong. To address this concern, the client keeps track of all speculative replies and piggybacks such replies with every speculative request. Upon receiving a speculative request, a server replica compares its logged replies with the piggybacked speculative replies. If the two do not match, the replica drops the speculative request because it knows that the client would have to be rolled back.

\subsection{Multi-Message Optimistic Fault Tolerance}

In multi-message optimistic fault tolerance, a replica executes a request as soon as it is received. The replica consistency is ensured by synchronizing the state of the replicas periodically, or on-demand whenever a conflict is detected. It is easy to understand that the checkpointing and redo mechanisms used for the recovery alone in single-message optimistic fault tolerance will not work for multi-message optimistic fault tolerance because the checkpoints 


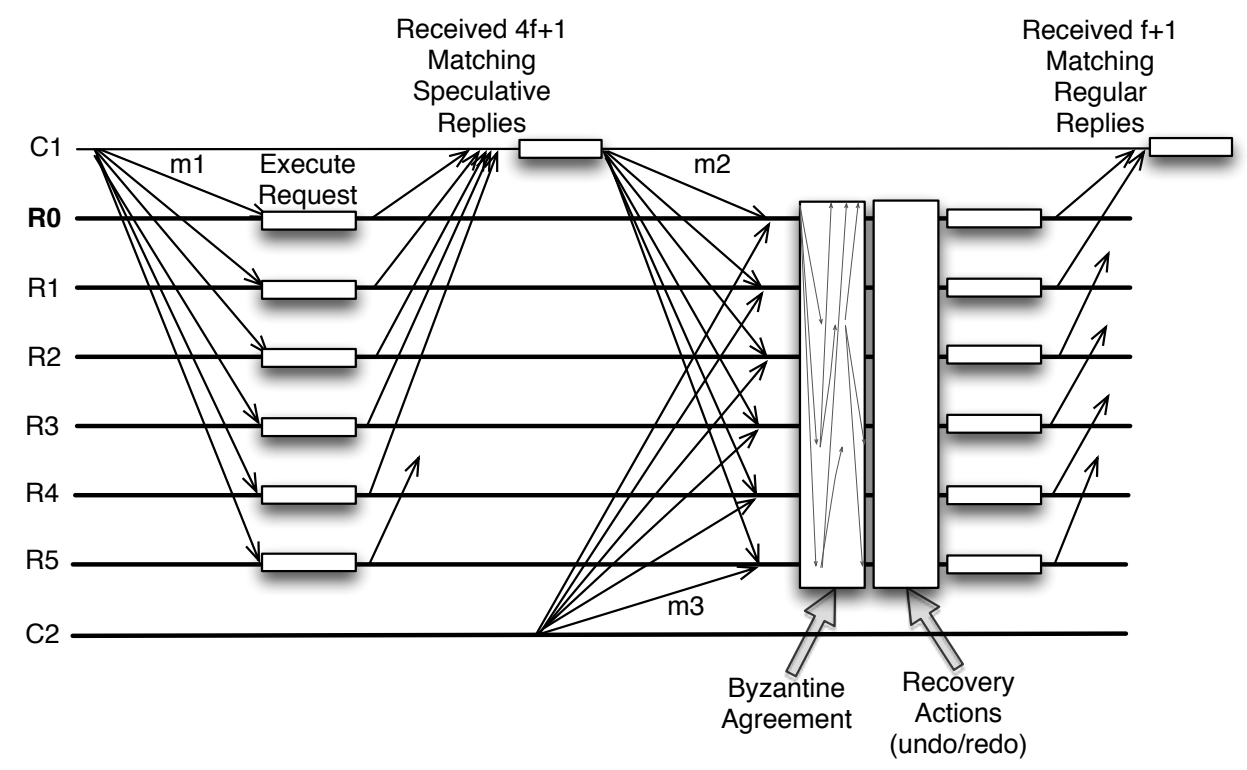

Figure 9: Two forms of operations in the replication algorithm for commutative requests.

produced at the synchronization point are guaranteed to be inconsistent if replicas have executed some requests in different orders.

\subsubsection{A General Purpose Replication Algorithm for Commutative Requests}

Raykov et al. proposed a general purpose algorithm (Raykov et al., 2011) that can support a mixture of commutative and non-commutative requests. The algorithm ensures that commutative requests are not totally ordered and it may reduce the end-to-end latency to as few as two communication steps for such requests. This algorithm requires the use of $5 f+1$ replicas when $f$ of them could be Byzantine faulty.

Figure 9 illustrates the operation of the algorithm. A client broadcasts its request to server replicas and waits until either one of the following conditions is satisfied:

- It has received $4 f+1$ matching speculative replies.

- It has received $f+1$ matching regular replies.

A server replica speculatively executes a request immediately if no other request that has been received but not yet delivered is conflicting with the current request, and a speculative reply is sent to the client. If a conflicting 
request is found, a recovery consensus phase is started to ensure the total ordering of conflicting requests. During the recovery consensus phase, each replica $i$ launches a Byzantine agreement instance on the following two sets of requests it has received in the current round and on the total ordering of the sets:

- NCSet ${ }_{i}$ : A set of requests that are not conflicting with each other (i.e., they are commutative) received by replica $i$.

- $C S_{e} t_{i}$ : A set of conflicting requests received by replica $i$. For any message $m$ in $C S e t_{i}$, there is at least one message $m^{\prime}$ in $N C S e t_{i}$ that is conflicting with $m$.

A replica $i$ waits until it has received $4 f+1$ sets of Byzantine agreed $N C S e t_{j}$ and $\mathrm{CSet}_{j}$ from different replicas to build a final set for commutative requests $N C S e t$ and the final set for conflicting requests $C S e t$, according to the following rules:

- If a message is present in the majority of $N C S e t_{j}$ collected, the message is included in NCSet.

- For all other messages in any of $N C S e t_{j}$ collected, as well as the messages in any $\mathrm{CSet}_{j}$ that are not included in the final NCSet, they are included in the final CSet.

The recovery consensus phase is needed to identify, and recover from, wrong speculations. In this phase, a request that is speculatively executed in the wrong order will be rolled back and re-executed in the correct total order. All conflicting requests are executed normally according to the total order established. A normal reply is sent to the client for each request that has been totally ordered.

\subsubsection{Application-Specific Optimistic Fault Tolerance}

As shown in Figure 10, three types of applications have been identified to fit optimistic fault tolerance well (Zhao, 2015) because their operations are either commutative, or can be rendered commutative via operational transformation (Ellis and Gibbs, 1989).

The first type is realtime collaborative editing applications (Sun et al., 1998). As we mentioned previously, this type of applications must allow different users to update a shared document concurrently, which makes it 


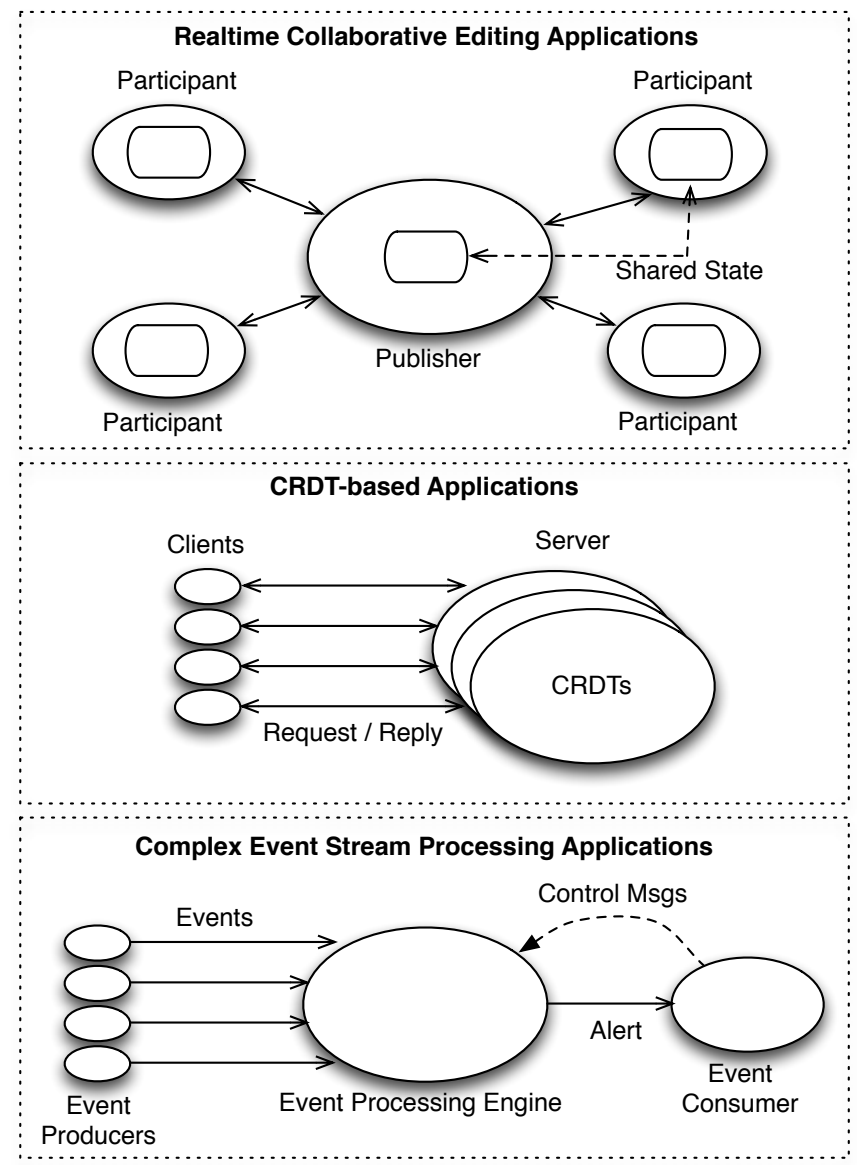

Figure 10: Three types of applications that fit optimistic fault tolerance well.

impossible to use conservative fault tolerance or single-message optimistic fault tolerance. For this type of applications under the crash-fault model, replica consistency can be achieved by using a vector timestamp to impose a total order on all requests, and by applying operational transformation (Ellis and Gibbs, 1989) to out-of-ordered requests. In the presence of Byzantine faults, this mechanism is insufficient to ensure replica consistency because the vector timestamp could be tempered with by a faulty replica. To cope with this challenge, during a round of state synchronization, replicas compare the list of requests that have been executed together with their vector timestamps. If a discrepancy is detected, the problematic requests are identified and undone, which is followed by a redo step with the correct order (Zhao 
and Babi, 2013).

The second type of applications includes those that are constructed with conflict-free (or commutative) replicated data types (CRDTs) (Shapiro et al., 2011). Even though all requests are commutative in this type of applications, and hence, their execution order is not important, state synchronization is still needed because different replicas might execute different sets of requests, which could lead to the divergence of replica state. The purpose of the state synchronization is to ensure that each replica executes the same set of requests, which achieves an eventual replica consistency.

To facilitate faster recovery, undo operations could be used to selectively cancel out the effect of the execution of some requests (Chai and Zhao, 2014a). An undo operation can be either provided by the application, or be constructed based on the application semantics. With the facility of undo operations, a replica can be brought to a desirable state from any inconsistent state by doing selective undos followed by redos, without the need of rolling back to a previous state.

The third type of applications consists of complex event stream processing applications (Etzion and Niblett, 2010). This type of applications typically generates alerts based on a group of events and events within the group are typically commutative. Hence, multi-message optimistic Byzantine fault tolerance is a good fit. Doing so also helps satisfy the soft-realtime requirement of such applications. Totally ordering all events clearly would not work because the runtime overhead for doing so might be too excessive for these applications.

For all three types of applications, in addition to periodic state synchronization, a round of on-demand state synchronization could be triggered on the detection of inconsistency among replicas by a client (Chai and Zhao, 2014b). The on-demand state synchronization closely works with the outputcommit mechanism. In a distributed system, when one component generates an output for another component, it must commit to the output, which is referred to output commit (Strom and Yemini, 1985). To satisfy this requirement, a client does not accept the output from a replicated component until it has received $2 f+1$ valid and matching outputs from the replicas. If the first $2 f+1$ messages received do not completely match, the client sets a timer and attempts to collect more output messages towards $2 f+1$ matching messages before the timer expires. When this attempt fails, the client sends a synchronization request to all replicas, which will trigger a round of on-demand state synchronization. 


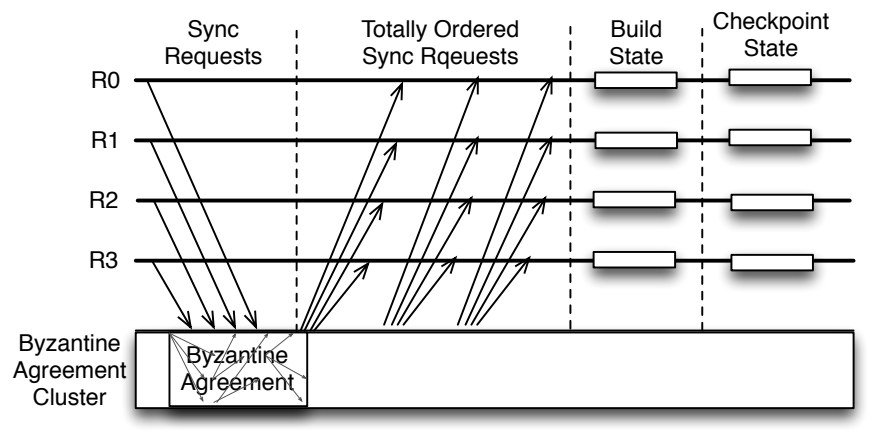

Figure 11: State synchronization using $2 f+1$ rounds of Byzantine agreement.

For both periodic state synchronization and on-demand state synchronization, an instance of Byzantine agreement on the state is involved. There are two approaches to accomplishing this task. In one approach (Zhang et al., 2012), this is done with an update phase followed by an instance of Byzantine agreement on the superset of records to be agreed upon by all nonfaulty replicas. Similar to what has been shown in Figure 7, during the update phase, all replicas broadcast their records to other replicas. Then, the primary replica builds a superset of the records collected during the update phase for Byzantine agreement. Although this approach is efficient in that only a single instance of Byzantine agreement is used in each state synchronization, it does depends on a particular replica (i.e., the primary) to propose a superset. If the primary is faulty, then a view change may be inevitable, which could delay the completion of the round of state synchronization.

An alternative approach is to use an external Byzantine agreement cluster and use $2 f+1$ instances of Byzantine agreement to synchronize the state without the update phase (Chai and Zhao, 2014a,b). In this approach, as shown in Figure 11, the superset (that represents the state to be synchronized) is built deterministically based on the identical set of totally ordered $2 f+1$ state messages at each replica. Hence, a faulty replica could not disrupt the superset formation as does in the former approach. As a tradeoff, $2 f$ additional instances of Byzantine agreement are needed.

\section{Future Research Directions}

While application semantics is essential for the design of practical fault tolerance systems, it may be costly to discover relevant application semantics. 
It may also be difficult to maintain the implementation of fault tolerance mechanisms when the application logic is changed.

These issues can be addressed in several fronts: (1) use structured data; (2) use standard, document-based remote communication interfaces; and (3) separate application logic execution from data management.

\subsection{Structured Data}

Structured data has long been used in transaction processing. In transaction processing, data types and operations on the data types in an application are predefined with one or more schemas (Gray and Reuter, 1992). As such, the state of an application and the operations on the state are readily available without the need of analyzing the source code. In addition to traditional transaction processing applications, general-purpose applications can be developed using software transactional memory (STM) (Shavit and Touitou, 1995), which offers many of the benefits of traditional transaction processing. A particular benefit of using transaction processing or STM is the elimination of manual thread synchronization, which is a source of major obstacle for developing highly concurrent fault tolerance systems. Structured data enables the adoption of well-defined concurrency control algorithms, which is also conducive to developing highly efficient fault tolerance solutions.

An exciting new research direction related to structured data is the use of CRDTs for developing fault tolerant applications (Shapiro et al., 2011). The use of CRDTs eliminates the need for totally ordering requests, hence, it could drastically reduce the number of distributed agreement instances needed in an application. A list of CRDTs are introduced in (Shapiro et al., 2011), including various types of counters, registers, sets, maps, graphs, and sequences. It is shown that these CRDTs can be used to build practical applications. In (Chai and Zhao, 2014a), the Two-Phase Set (2P-Set) was used to implement a shopping cart service. A 2P-Set consists of two grow-only sets, one for adding objects to the shopping cart, the other for removing objects from the shopping cart. The latter set is often referred to as the tombstone set. The 2P-Set ensures that the "add" and "remove" operations on the same shopping cart are commutative (the "add" and "remove" operations on different shopping carts are apparently commutative).

In both cases, the use of structured data also makes it easier to develop undo operations, which are essential to enable fast state synchronization in optimistic fault tolerance. 


\subsection{Standard Document-Based Interfaces}

Document-based remote communication interfaces facilitate the development of loosely coupled distributed systems, which by itself is conducive for fault tolerance. If the interface conforms to standards, such as various Web services standards (Little and Wilkinson, 2007; Freund and Little, 2007; Feingold and Jeyaraman, 2007), the interactions between different components of an application are predefined in an interface file. Hence, the application semantics can be discovered without cumbersome source code analysis or expert knowledge.

\subsection{Separation of Application Logic from Data Management}

The separation of application logic execution from data management, which is popular in many Web-based applications (often reflected via the three-tier architecture), enables the use of stateless design in application servers running at the middle tier, with persistent data stored by dedicated database management systems (Zhao et al., 2005b; Zhao, 2014c). As shown in (Chai and Zhao, 2013), such systems can be rendered Byzantine fault tolerant without requiring deep knowledge of application semantics.

\section{Conclusion}

In this article, we presented an overview of various performance engineering techniques for practical fault tolerance systems based on state machine replication. Most such techniques depend on the integration of application semantics into fault tolerance design. We first elaborated that application semantics is not only needed for achieving better performance, but for ensuring the correctness of the systems as well. We then provided a classification of these approaches, which is followed by the description of the mechanisms used in these approaches. Finally, we outlined potential future research directions related to performance engineering of fault tolerance systems. When

designing a distributed system for fault tolerance, we advocate the use of structured data and standard, document-based remote communication interfaces, and the separation of application logic from data management. For such systems, it is easier to discover application semantics and maintain fault tolerance solutions. 


\section{Acknowledgements}

I sincerely thank the reviewers for their invaluable suggestions on how to improve an earlier version of this article. This work was supported in part by NSF grant CNS-0821319 and by a graduate faculty travel award from Cleveland State University.

\section{References}

Adya, A., Bolosky, W. J., Castro, M., Cermak, G., Chaiken, R., Douceur, J. R., Howell, J., Lorch, J. R., Theimer, M., Wattenhofer, R. P., 2002. Farsite: Federated, available, and reliable storage for an incompletely trusted environment. In: Proceedings of the 5th Symposium on Operating Systems Design and Implementation. pp. 1-14.

Birman, K. P., 1985. Replication and fault-tolerance in the isis system. In: Proceedings of the 10th ACM Symposium on Operating Systems Principles. ACM, pp. 79-86.

Brewer, E. A., 2012. Pushing the cap: Strategies for consistency and availability. IEEE Computer 45 (2), 23-29.

Brito, A., Fetzer, C., Felber, P., 2009. Multithreading-enabled active replication for event stream processing operators. In: Proceedings of the 28th IEEE International Symposium on Reliable Distributed Systems. IEEE, pp. $22-31$.

Burrows, M., 2006. The chubby lock service for loosely-coupled distributed systems. In: Proceedings of the 7th symposium on Operating systems design and implementation. USENIX Association, Berkeley, CA, USA, pp. $335-350$.

Castro, M., Liskov, B., 2002. Practical byzantine fault tolerance and proactive recovery. ACM Transactions on Computer Systems 20 (4), 398-461.

Castro, M., Rodrigues, R., Liskov, B., 2003. Base: Using abstraction to improve fault tolerance. ACM Transactions on Computer Systems 21 (3), 236-269. 
Chai, H., Zhang, H., Zhao, W., Melliar-Smith, P. M., Moser, L. E., 2013. Toward trustworthy coordination for web service business activities. IEEE Transactions on Services Computing 6 (2), 276-288.

Chai, H., Zhao, W., 2012a. Byzantine fault tolerance as a service. In: Kim, T.-h., Mohammed, S., Ramos, C., Abawajy, J., Kang, B.-H., Slezak, D. (Eds.), Computer Applications for Web, Human Computer Interaction, Signal and Image Processing, and Pattern Recognition. Vol. 342 of Communications in Computer and Information Science. Springer Berlin Heidelberg, pp. 173-179.

Chai, H., Zhao, W., 2012b. Interaction patterns for byzantine fault tolerance computing. In: Kim, T.-h., Mohammed, S., Ramos, C., Abawajy, J., Kang, B.-H., Slezak, D. (Eds.), Computer Applications for Web, Human Computer Interaction, Signal and Image Processing, and Pattern Recognition. Vol. 342 of Communications in Computer and Information Science. Springer Berlin Heidelberg, pp. 180-188.

Chai, H., Zhao, W., 2013. Byzantine fault tolerance for session-oriented multi-tiered applications. Int. J. of Web Science 2 (1/2), 113-125.

Chai, H., Zhao, W., June 27 - July 2 2014a. Byzantine fault tolerance for services with commutative operations. In: Proceedings of the IEEE International Conference on Services Computing. IEEE, Anchorage, Alaska, USA, pp. 219-226.

Chai, H., Zhao, W., 2014b. Byzantine fault tolerant event stream processing for autonomic computing. In: Proceedings of the 12th IEEE International Conference on Dependable, Autonomic and Secure Computing. IEEE, pp. 109-114.

Chai, H., Zhao, W., 2014c. Towards trustworthy complex event processing. In: Proceedings of the 5th IEEE International Conference on Software Engineering and Service Science. IEEE, pp. 758-761.

Chandra, T. D., Griesemer, R., Redstone, J., 2007. Paxos made live: an engineering perspective. In: Proceedings of the twenty-sixth annual ACM symposium on Principles of distributed computing. PODC '07. ACM, New York, NY, USA, pp. 398-407. 
Clement, A., Kapritsos, M., Lee, S., Wang, Y., Alvisi, L., Dahlin, M., Riche, T., 2009. Upright cluster services. In: Proceedings of the ACM symposium on Operating systems principles. ACM, New York, NY, USA, pp. 277-290.

Cowling, J., Myers, D., Liskov, B., Rodrigues, R., Shrira, L., 2006. Hq replication: A hybrid quorum protocol for byzantine fault tolerance. In: Proceedings of the 7th symposium on Operating systems design and implementation. USENIX Association, pp. 177-190.

Ellis, C. A., Gibbs, S. J., 1989. Concurrency control in groupware systems. In: Proceedings of the ACM SIGMOD international conference on Management of data. ACM, New York, NY, USA, pp. 399-407.

Etzion, O., Niblett, P., 2010. Event Processing in Action. Manning Publications.

Feingold, M., Jeyaraman, R., July 2007. Web services coordination, version 1.1, OASIS standard.

Felber, P., 2001. Lightweight fault tolerance in corba. In: Proceedings of the 3rd International Symposium on Distributed Objects and Applications. IEEE, pp. 239-247.

Freund, T., Little, M., April 2007. Web Services Business Activity Version 1.1, OASIS standard.

Gray, C., Cheriton, D., 1989. Leases: an efficient fault-tolerant mechanism for distributed file cache consistency. In: Proceedings of the 12th ACM symposium on Operating systems principles. ACM, New York, NY, USA, pp. 202-210.

Gray, J., Reuter, A., 1992. Transaction Processing: Concepts and Techniques, 1st Edition. Morgan Kaufmann Publishers Inc., San Francisco, CA, USA.

Kotla, R., Alvisi, L., Dahlin, M., Clement, A., Wong, E., 2009. Zyzzyva: Speculative byzantine fault tolerance. ACM Transactions on Computer Systems (TOCS) 27 (4), 7.

Kotla, R., Dahlin, M., 2004. High throughput byzantine fault tolerance. In: Proceedings of International Conference on Dependable Systems and Networks. pp. 575-584. 
Lamport, L., 1998. The part-time parliament. ACM Transactions on Computer Systems 16 (2), 133-169.

Lamport, L., December 2001. Paxos made simple. ACM SIGACT News (Distributed Computing Column) 32 (4), 18-25.

Li, C., Porto, D., Clement, A., Gehrke, J., Preguiça, N. M., Rodrigues, R., October 2012. Making geo-replicated systems fast as possible, consistent when necessary. In: Proceedings of the 10th USENIX Symposium on Operating Systems Design and Implementation. Hollywood, CA, pp. 265-278.

Little, M., Wilkinson, A., April 2007. Web Services Atomic Transactions Version 1.1, OASIS standard.

MacCormick, J., Murphy, N., Najork, M., Thekkath, C. A., Zhou, L., 2004. Boxwood: Abstractions as the foundation for storage infrastructure. In: Proceedings of the USENIX Symposium on Operating Systems Design and Implementation. pp. 105-120.

Malkhi, D., Reiter, M., 1998. Byzantine quorum systems. Distributed Computing 11 (4), 203-213.

Moraru, I., Andersen, D. G., Kaminsky, M., 2013. There is more consensus in egalitarian parliaments. In: Proceedings of the Twenty-Fourth ACM Symposium on Operating Systems Principles. ACM, pp. 358-372.

Moser, L. E., Melliar-Smith, P. M., Agarwal, D. A., Budhia, R. K., LingleyPapadopoulos, C. A., Apr. 1996. Totem: a fault-tolerant multicast group communication system. Commun. ACM 39 (4), 54-63.

URL http://doi .acm.org/10.1145/227210.227226

Ramakrishnan, R., 2012. Cap and cloud data management. IEEE Computer 45 (2), 43-49.

Raykov, P., Schiper, N., Pedone, F., 2011. Byzantine fault-tolerance with commutative commands. In: Fernndez Anta, A., Lipari, G., Roy, M. (Eds.), Principles of Distributed Systems. Vol. 7109 of Lecture Notes in Computer Science. Springer Berlin Heidelberg, pp. 329-342.

Saito, Y., Shapiro, M., Mar. 2005. Optimistic replication. ACM Comput. Surv. 37 (1), 42-81. 
Shapiro, M., Preguia, N., Baquero, C., Zawirski, M., 2011. Conflict-free replicated data types. In: Dfago, X., Petit, F., Villain, V. (Eds.), Stabilization, Safety, and Security of Distributed Systems. Vol. 6976 of Lecture Notes in Computer Science. Springer Berlin Heidelberg, pp. 386-400.

Shavit, N., Touitou, D., 1995. Software transactional memory. In: Proceedings of the 14th ACM Symposium on Principles of Distributed Computing. pp. 204-213.

Strom, R., Yemini, S., Aug. 1985. Optimistic recovery in distributed systems. ACM Trans. Comput. Syst. 3 (3), 204-226.

Sun, C., Jia, X., Zhang, Y., Yang, Y., Chen, D., Mar. 1998. Achieving convergence, causality preservation, and intention preservation in real-time cooperative editing systems. ACM Trans. Comput.-Hum. Interact. 5 (1), 63-108.

Wester, B., Cowling, J. A., Nightingale, E. B., Chen, P. M., Flinn, J., Liskov, B., 2009. Tolerating latency in replicated state machines through client speculation. In: Proceedings of the Networked Systems Design and Implementation. pp. 245-260.

Yin, J., Martin, J. P., Venkataramani, A., Alvisi, L., Dahlin, M., 2003. Separating agreement from execution for byzantine fault tolerant services. In: Proceedings of the ACM Symposium on Operating Systems Principles. Bolton Landing, NY, pp. 253-267.

Zhang, H., Chai, H., Zhao, W., Melliar-Smith, P. M., Moser, L. E., 2012. Trustworthy coordination for web service atomic transactions. IEEE Transactions on Parallel and Distributed Systems 23 (8), 1551-1565.

Zhang, H., Zhao, W., 2012. Concurrent byzantine fault tolerance for softwaretransactional-memory based applications. International Journal of Future Computer and Communication 1 (1), 47-50.

Zhang, H., Zhao, W., Melliar-Smith, P. M., Moser, L. E., 2011. Design and implementation of a byzantine fault tolerance framework for nondeterministic applications. IET Software 5, 342-356. 
Zhao, W., December 2008. Integrity-preserving replica coordination for byzantine fault tolerant systems. In: Proceedings of the IEEE International Conference on Parallel and Distributed Systems. Melbourne, Victoria, Australia, pp. 447-454.

Zhao, W., June 2009. Design and implementation of a Byzantine fault tolerance framework for web services. Journal of Systems and Software 82 (6), $1004-1015$.

Zhao, W., 2014a. Application-aware byzantine fault tolerance. In: Proceedings of the 12th IEEE International Conference on Dependable, Autonomic and Secure Computing. IEEE, pp. 45-50.

Zhao, W., 2014b. Building Dependable Distributed Systems. WileyScrivener.

Zhao, W., 2014c. A novel approach to building intrusion tolerant systems. International Journal of Performability Engineering 10 (2), 123.

Zhao, W., 2015. Optimistic byzantine fault tolerance. International Journal of Parallel, Emergent and Distributed Systems (ahead-of-print), 1-14.

Zhao, W., Babi, M., 2013. Byzantine fault tolerant collaborative editing. In: Proceedings of the IET International Conference on Information and Communications Technologies. IET, pp. 233-240.

Zhao, W., Melliar-Smith, P. M., Moser, L. E., 2013. Low latency fault tolerance system. The Computer Journal 56 (6), 716-740.

Zhao, W., Moser, L., Melliar-Smith, P. M., 2005a. Deterministic scheduling for multithreaded replicas. In: Proceedings of the IEEE International Workshop on Object-oriented Real-time Dependable Systems. Sedona, AZ, pp. $74-81$.

Zhao, W., Moser, L. E., Melliar-Smith, P. M., 2005b. Unification of transactions and replication in three-tier architectures based on CORBA. IEEE Transactions on Dependable and Secure Computing 2 (1), 20-33.

Zhao, W., Zhang, H., Chai, H., 2009. A lightweight fault tolerance framework for web services. Web Intelligence and Agent Systems 7, 255-268. 